\title{
Fault diagnosis of compressor based on decision tree and fuzzy inference system
}

\author{
Milad Golmoradi ${ }^{1}$, Ebrahim Ebrahimi², Mohamad Javidan ${ }^{3}$ \\ Department of Mechanical Engineering, College of Engineering, Kermanshah Branch, \\ Islamic Azad University, Kermanshah, Iran \\ ${ }^{1}$ Corresponding author \\ E-mail: 19olmoradi17@gmail.com, ${ }^{2}$ e.ebrahimi57@gmail.com, ${ }^{3}$ javidan833@gmail.com
}

Received 27 March 2017; accepted 13 April 2017

DOI https://doi.org/10.21595/vp.2017.18398

Check for updates

\begin{abstract}
There is a strong need to use different methods for maintaining high product quality and safety production line, given the rapid technological progress. Thus, the condition monitoring is widely used as an efficient method in various industries. A variety of methods have been used so far in order to implement condition monitoring system, the most common one has been seismic waves analysis in the time-frequency domain. The current paper proposes a Fuzzy Inference System for monitoring the status of the compressor based on Daubechies wavelet transform and decision trees. The $\mathrm{J} 48$ algorithm was used as a tool for classification design and selection of effective features on troubleshooting. The J48 algorithm output results showed that signal processing technique by Daubechies wavelet mother has the highest accuracy for the implementation of the FIS system. The J48 algorithm output is a decision tree used on production of if-then rules and fuzzy set membership functions of the system. Finally, the combination of WT-J48-FIS overall accuracy for classifying compressor defects of $93.33 \%$ was obtained.
\end{abstract}

Keywords: compressor, signal processing, vibrations analysis, decision tree, FIS.

\section{Introduction}

As an efficient and effective method for improving factors of confidence in and health and optimal performance of machineries, condition monitoring has drawn a lot of attention to itself. In addition, hitherto numerous studies concerning condition monitoring and troubleshooting of machineries have been conducted and many papers have been published on this subject. Condition monitoring signifies troubleshooting and maintenance of equipment without stopping them from running [1]. In general, these method is based on orderly recording of data of dynamic characteristics of equipment and comparing with control healthy state. In classic condition monitoring, troubleshooting is usually done based on vibrations and/or sound data [2]. Numerous methods developed for single-sensor condition monitoring are premised on a definite characteristic such as vibrations [3,4] and sound [5] and based on a certain classifier such as support vector machine [6], artificial neural network [7], and fuzzy logic [8]. Compressors are one of the most common devices used in current industries. The problem of increased pressure in different industrial processes, especial petrochemical ones, is highly significant. Compressor is a device used for increasing pressure of gas and transferring it from one point to another during the process. This pressure is required for dominating over friction in the path, effect on a definite reaction and improving thermodynamic characteristics of a gas. There are numerous industries and fields in which compressor plays a critical role such as ventilation of buildings, compressed air supply to the combustion in internal combustion engines, gas transmission, pressure supply for storage tanks, and injection of gas into oil reservoirs. As a result of periodical instability of airflow of compressor, resonance occurs and finally, the blade cracks and fractures due to high-cycle fatigue [9]. Contact between blade and root of the blade gradually wears and weakens the blade. The contact contributes to cracking and failure of the blade. Crack at the point of connection between disk to shaft due to fatigue [10], crack at point of connection between blade and disk under influence of fatigue [11] and uncommon loading are main factors contributing to fracturing and cracking of blades [12]. The results suggested that fractures and cracks of blades are result of 
resonance and high-cycle fatigue of the blade. The latter event could be result of serious disturbances in air flow of compressor, instability of flow, and weakened materials used in the blade and under influence of pitting corrosion and fatigue corrosion. Therefore, troubleshooting of compressors is significant in terms of prevention from larger fractures and failure. This increasing significance has drawn the attention of many researchers to condition monitoring of compressors. Most failures of compressors are found at their blades. Rama Rao and Duttam (2012) used vibrations analysis to analyze failures of blade of compressor [13]. In the study, they examined effects of vibrational modes on intensity of failures found in blades. In the study, they found failures in 5 blades of compressors and found out that these failures are visible in vibrational mode of $508 \mathrm{~Hz}$. Fujiwara and Sakurai (1986) analyzed noise and vibration of rotary positive displacement compressors with spiral-shaped piston in laboratory setting [14]. They studies results of modal analysis and dynamic responses under different conditions.

\section{Materials and methods}

The primary objective of present study is smart detection of compressor in gas industry through processing vibrations generated by a system. In this project, item to be tested was a gas compressor located in a gas compressor station of Iranian Gas Transmission Company. Fig. 1 shows a scheme of compressor.

\subsection{Fault analysis}

The most critical failures of compressors occur in their blades. Consequently, cracks and fractures of blades of compressors belonging to Iran-Arak Gas Transmission Company were analyzed in present study. If detection system is developed properly, detection of small failures will be possible too.

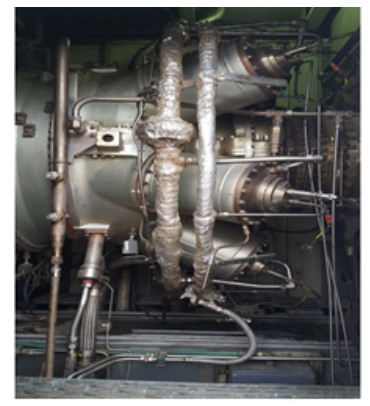

a)

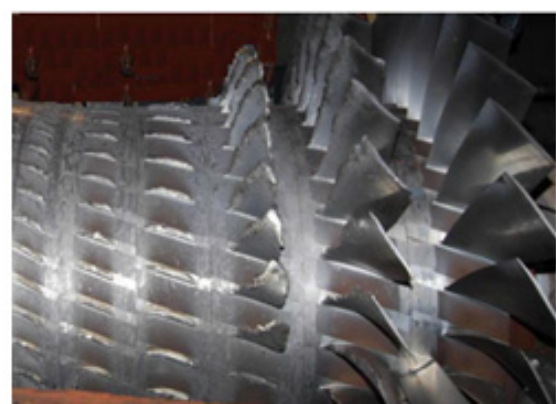

b)

Fig. 1. a) Ten-step compressor, b) fracture and wear of compressor blades

\subsection{Signal processing}

Considering the fact that compressors work at high cycles, generated vibrations and fatigues are factors contributing to fracture and failure of these sets. In normal operating conditions, compressor has an allowed range of vibrations. If these vibrations exceed a definite limit, it suggests that compressor is no longer operating in normal conditions. On the other hand, each part or element of compressor runs at a certain frequency. If a failure occurs in this set, it is represented at a distinct frequency and range of vibrations. Therefore, the present study uses vibration signals for condition monitoring of compressors [10]. Before analysis of signals in pre-processing step, samples were imported into MATLAB Software and wavelet transform of each sample which represents distinctive states of compressor was done. As Fig. 2 suggests, signals collected by sensors in the first step were wholly at time domain. Although vibration data offers valuable information on condition of the machine, but such data does not include failure signal and there 
are numerous noises in them. The noises in time-domain signals problematize direct detection of failures [4]. Addressing this problem requires transfer of signals from time domain to timefrequency domain. In this case, noise is excluded and more useful information could be obtained. There are numerous methods for signal processing among which one could point to wavelet transform.

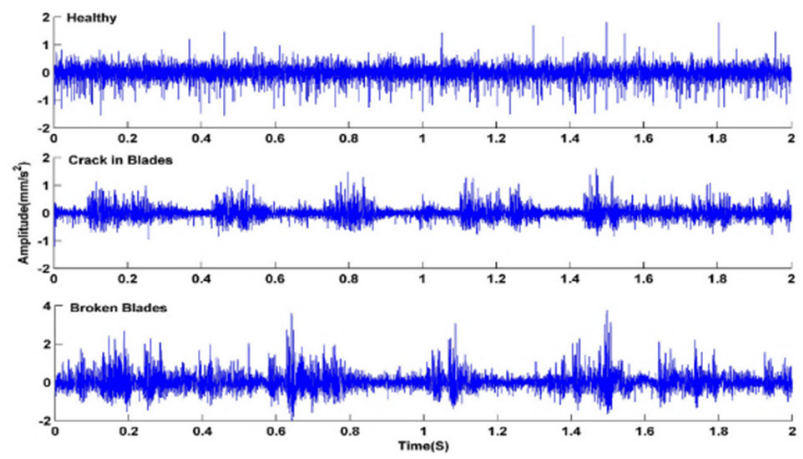

Fig. 2. Vibration signals in different states of compressor

\subsection{Vibration spectra feature extraction}

Direct use of wavelet transfer outputs in smart systems is not possible. Consequently, certain functions should be used for computation of initial signal features. Statistical features and some functions used in condition monitoring used for extraction of features include mean F1, standard deviations F2, mean squared error F3, Skewness F4, variance F5, kurtosis F6, geometric mean F7, steepness factor F8, harmonic mean F9, and slip F10.

\subsection{Smart troubleshooting system}

\subsubsection{Decision tree}

In 1963, decision tree method was initially introduced by Morgan and Sunkist. Decision tree is one of the most significant techniques in data mining. As a part of data-mining systems, decision trees act as a powerful tool for classification.

\subsubsection{C4.5 algorithm}

In the present study, classification of compressor failures through features extracted from vibration spectra of failures was done via Weka Software and J48 algorithm. Weka is a set of machine learning algorithms and pre-processing tools. A specific label was assigned to different states of compressor so as to classify failures (Healthy = Compressor in a healthy condition, Broken-Blades $=$ breakage in blades, Crack in Blades $=$ Crack in Blades). From each compressor states, 50 spectra were obtained. Then, in a completely random manner 70 percent of each trait was used for training and extraction of classifier while remaining 30 percent was used for final measurement of fuzzy model.

\subsection{Results of data-mining}

The results of output of J48 algorithm are shown in Table 1. In this case, R-values suggest accuracy of classification for different classes. In each mode of mother wavelet, accuracy of algorithm $\mathrm{J} 48$ in detection of different classes is different. Use of mother wavelet $\mathrm{Db} 3$ for processing of vibration signals offered best precision compared with other states.

In this regard, implementation of fuzzy system based on decision tree of data processing was 
premised on Daubechies wavelet transform. The other two methods offer less precision in implementation of fuzzy system so that values of table 1 suggest that other mother wavelets show less precision in classification of failures.

Fig. 3 shows decision tree for output of algorithm J48 and case of signal processing through Db3 wavelet transform. Tracing a branch from main node to leaf leads to a state of compressor and decryption of information in each tree branch as if-then sentences develops essential regulation for fuzzy classification of failures of compressor. Evidently, for each tree the top node is best node for classification. Other features in nodes of decision tree are arranged based on decreasing significance. In decision trees, a category of spectrum features is placed which is significant for correct classification. Those features that lack essential significance for classifying failures of compressor are excluded from the model. Data distribution level for set of features that compose a class is represented by in-parenthesis numbers within decision tree and before condition of compressor at that class. In this case, the first number in parenthesis suggests number of data which could be classified properly through a set of features. The second number refers to number of data that are incorrectly placed in that class. If first number in parenthesis is much smaller than all data included in the model to suggest a failure, that class could be excluded from final model.

Table 1. Comparison of results of data training step in application of algorithm J48

\begin{tabular}{|c|c|c|}
\hline Type of analysis & $R$ (Kappa statistic) & RMSE \\
\hline WT-Coif3 & 0.9586 & 0.0828 \\
\hline WT-Sym3 & 0.9643 & 0.1069 \\
\hline WT-Db3 & 0.9710 & 0.0478 \\
\hline
\end{tabular}

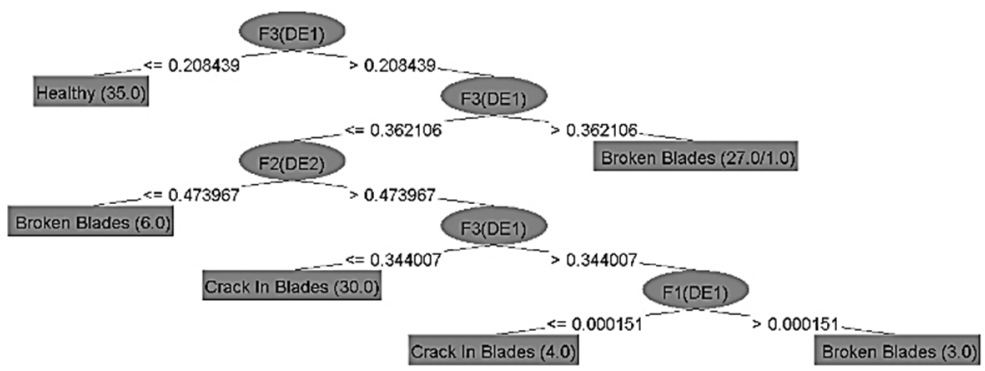

Fig. 3. Decision tree

\subsection{Implementation of fuzzy inference system (FIS)}

\subsubsection{Membership functions}

Considering the fact that each branch of decision tree represents a certain condition of compressor, membership functions were developed for statistical cases of signal analysis in MATLAB Software. There is a numerical threshold for each membership function. For lower values than interface, membership function generates a zero. For higher values than threshold, membership function generates a "one". A membership function with such behavior is called trapezoidal membership function. In present study, trapezoidal membership function was used for representation of all points in input space. Fig. 4 shows membership functions of the compressor based on decision-tree.

Outputs of fuzzy classifier are those conditions of compressor that had been labelled healthy, broken blades, and crack in clades. Therefore, there are three outputs for fuzzy classifier and for them, three trapezoidal membership functions with equal range from 0 to 3 were developed. As shown in Fig. 4, the range 0-1 refers to healthy condition, range 1-2 refers to crack in blades, and range 2-3 refers to broken blades. 


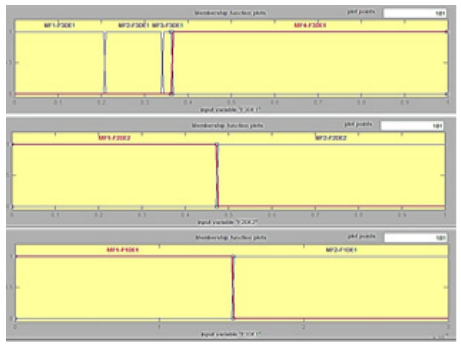

a)

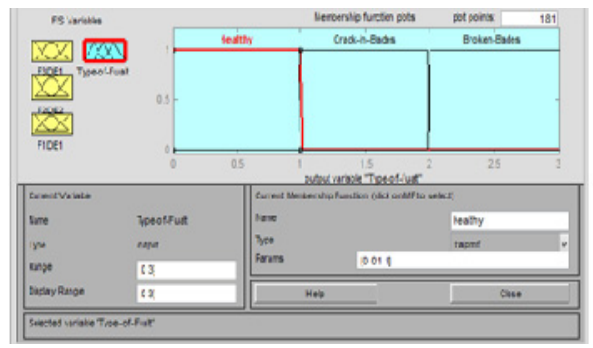

b)

Fig. 4. a) Membership function for features F3De1, F2De2 and F1De1,

b) membership functions for fuzzy output

\subsubsection{Fuzzy rules}

Fuzzy rules were developed as if-then through decision tree. In all of these rules, order of rules is not significant and all of rules are of equal level of significance. In the following, these rules are detailed:

If (F3DE1 is MF1-F3DE1) then (Type-of-Fault is Healthy) (1).

If (F3DE1 is MF2-F3DE1) and (F2DE2 is MF1-F2DE2) then (Type-of-Fault is Broken-Blades) (1).

If (F3DE1 is MF2-F3DE1) and (F2DE2 is MF2-F2DE2) then (Type-of-Fault is Crack-in-Blades) (1).

If (F3DE1 is MF3-F3DE1) and (F2DE2 is MF2-F2DE2) and (F1DE1 is MF1-F1DE1) then (Type-of-Fault is Crack-in-Blades) (1).

If (F3DE1 is MF3-F3DE1) and (F2DE2 is MF2-F2DE2) and (F1DE1 is MF2-F1DE1) then (Type-of-Fault is Broken-Blades) (1).

If (F3DE1 is MF4-F3DE1) then (Type-of-Fault is Broken-Blades) (1).

\subsubsection{Fuzzy output}

The outputs of developed system could be viewed in Rule Viewer Editor. The editor is shown in Fig. 5. In this figure, each row corresponds with a certain condition of compressor. Each column represents inputs F3De1, F2De2 and F1De1. The fourth column is correlated with membership functions developed for fuzzy output. By moving a slipper on blocks of each input membership functions, one could obtain different outputs. With help of input samples for F3De1, F2De2 and F1De1, the rules were tested in the following manner. For input values, F2D2 was equal with 0.01 , F3De1 was equal with 0.05 and F2De 2 was equivalent with 0.00015 which satisfy the rules. The correlated output for it was healthy condition.

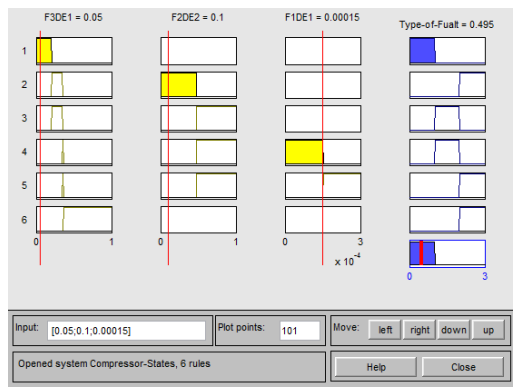

Fig. 5. Rule viewer developed for a sample of data

Performance of the system was calculated through some statistical parameters such as sensitivity and degree of compliance of total classification. The definitions of these statistical 
parameters are as defined in the following manner [7].

Sensitivity is number of positive and correct decisions in each condition of compressor divided by total number of decisions in that condition.

Degree of compliance of general classification is number of correct decisions divided by total number of possible decisions.

\section{Results}

In data collection step, vibrations data is measured through a piezoelectric accelerometer. Each one of three classes studied in this study has 50 vibration samples and in sum, there were 150 samples. Data of each class is divided into two sections. It should also be noted that 35 samples were randomly selected for training and another 15 samples were used for testing performance of troubleshooting system. The confusion matrix resulting from test of final model is represented in Table 2. The values of statistical parameters used for final evaluation based on confusion matrix are represented in Table 3. In the case of comparing variation range in vibration signals of different conditions of compressor, distinction between failed compressor and healthy compressor is easy. In regard to classification of failures of compressor, decision trees contribute to ease and simplicity of developing high-precision fuzzy interference system since decision trees are structurally simple and they could develop fuzzy rules and threshold values of membership functions. The results suggested that combined system FIS-J48 could distinguish healthy condition of compressor from its failed condition at a precision level of 100 percent. Detection of cracks on blades is due to intermediate condition between break of blade and health of the blade since combined method shows higher error in detection of crack than other conditions. In addition, total precision of system in detection of failures was reported to be 93.33 percent and this suggests an acceptable method for condition control of compressor. Consequently, one could state that this system is able to develop a precise detection of failures of compressor at 93 percent of cases. In addition, results of this design could be extended to other rotating mechanical systems. To do this, decision tree could be retrained with new training data and implement fuzzy system on it. Review of results of present study with those of other studies suggest that the method is an acceptable one for monitoring condition of industrial compressors.

Table 2. Confusion matrices of testing final model for different states of signal analysis

\begin{tabular}{|c|c|c|c|}
\hline Classification labelling & Healthy & Crack in blades & Broken blades \\
\hline Healthy & 15 & 0 & 0 \\
\hline Crack in blades & 1 & 13 & 1 \\
\hline Broken blades & 0 & 1 & 14 \\
\hline
\end{tabular}

Table 3. Statistical parameters of system evaluation for different cases of signal analysis

\begin{tabular}{|c|c|c|}
\hline $\begin{array}{c}\text { Evaluation parameter (\%) } \\
\text { classification label }\end{array}$ & Sensitivity & $\begin{array}{c}\text { Degree of compliance } \\
\text { of total classification }\end{array}$ \\
\hline Healthy & 100 & \multirow{2}{*}{93.33} \\
\hline Crack in blades & 86.67 & \\
\hline Broken blades & 93.33 & \\
\hline
\end{tabular}

\section{Conclusions}

The primary objective of present study is smart detection of multiple failure of compressors quickly and at high level of precision. Statistical features were extracted from time-frequency domain so as to detect different failures of compressors. In present survey, a method was introduced for condition monitoring of compressor based on fuzzy Inference System (FIS) as well as decision trees. The algorithm J48 was used as a method for developing decision tree and acted as an instrument for designing of classifier and selection of features affecting failure detection. Results of evaluating FIS model through experimental data suggested that total precision of 
classification is 93.33 percent. The obtained results pointed to applicability and effectiveness of this method for detection of failures of compressor.

\section{References}

[1] Ming Yang V. M. ARX model-based gearbox fault detection and localization under varying load conditions. Sound and Vibration, Vol. 329, Issue 24, 2010, p. 5209-5221.

[2] Miao Q. V. M. Condition monitoring and classification of rotating machinery using wavelets and hidden Markov models. Mechanical Systems and Signal Processing, Vol. 21, Issue 2, 2007, p. $840-855$.

[3] Niu G., Han T., Yang B. S., Andy C. C. T. Multi-agent decision fusion for motor fault diagnosis. Mechanical Systems and Signal Processing, Vol. 21, Issue 3, 2007, p. 1285-1299.

[4] Wang X., Makis V., Yang M. A wavelet approach to fault diagnosis of a gearbox under varying load conditions. Sound and Vibration, Vol. 329, Issue 9, 2010, p. 1570-1585.

[5] Bagheri B., Ahmadi H., Labbafi R. Implementing discrete wavelet and artificial neural networks for acoustic condition monitoring of gearbox. Elixir Mechanical Engineering, Vol. 35, 2011, p. 2909-2911.

[6] Yuan S., Chu F. Fault diagnosis based on support vector machines with parameter optimisation by artificial immunisation algorithm. Mechanical Systems and Signal, Vol. 21, Issue 3, 2007, p. $1318-1330$.

[7] Farokhzad S., Ahmadi H., Jaefari A., Asadi Asad Abad M. R., Ranjbar M. Artificial neural network based classification of faults in centrifugal pump. Journal of Vibroengineering, Vol. 14, Issue 4, 2012, p. 1734-1744.

[8] Mollazade K., Ahmadi H., Omid M., Alimardani R. An intelligent combined method based on power spectral density, decision trees and fuzzy logic for hydraulic pumps fault diagnosis. World Academy of Science, Engineering and Technology, Vol. 44, 2008, p. 895-907.

[9] Pursaidi A., Babai A., Jamshidi P., Behruzshad F. The study of reasons behind fracture of blades of compressors used in GE Frame 6 turbines based on vibrations analysis approach and CFD. 4th Conference of Power Plants, Power and Electronic Engineering Forum, Tehran, Iran, 2011.

[10] Farrahi G. H., Tirehdast M., Masoumi Khalil Abad E., Parsa S., Motakefpoor M. Failure analysis of a gas turbine compressor. Engineering Failure Analysis, Vol. 18, Issue 1, 2011, p. 474-484.

[11] Torabi A. R., Bayati M. Failure Analysis and development of a new solution for repairing disk of compressor in a gas turbine. 4th Conference of Power Plants, Power and Electronic Engineering Forum, Tehran, Iran, 2011.

[12] Torabi A. R., Bayati M. Failure analysis and repair of a catastrophically damaged gas turbine compressor disk using SEM technique and CFD Analysis. Journal of Failure Analysis and Prevention, Vol. 12, Issue 4, 2012, p. 391-401.

[13] Rama Rao A., Dutta B. K. Vibration analysis for detecting failure of compressor blade. Engineering Failure Analysis, Vol. 25, 2012, p. 211-218.

[14] Fujiwara A., Sakurai N. Experimental analysis of screw compressor noise and vibration. International Compressor Engineering Conference, 1986. 